\title{
Internacionalização da produção científica em Ciências Biológicas da UFRGS: 2000-20111
}

\author{
Internacionalization of scientific production in \\ Biological Sciences at UFRGS: 2000-2011
}

\author{
Dirce Maria SANTIN² \\ Samile Andrea de Souza VANZ3 \\ Ida Regina Chittó STUMPF³
}

\section{Resumo}

O artigo analisa a internacionalização da produção científica em Ciências Biológicas da Universidade Federal do Rio Grande do Sul, publicada na forma de artigos no período de 2000 a 2011, em periódicos indexados pela Web of Science. A pesquisa constitui-se num estudo bibliométrico, e a internacionalização é examinada sob três dimensões principais: difusão, colaboração e impacto internacional. Os indicadores de difusão internacional demonstram forte preferência pelos periódicos estrangeiros e pelo idioma inglês. A publicação dos artigos ocorreu em periódicos provenientes de 37 países, e a proporção de documentos veiculados em periódicos estrangeiros foi de $81,9 \%$. Os indicadores de colaboração internacional revelam a predominância da coautoria na publicação dos resultados das pesquisas. A colaboração internacional ocorreu em 22,4\% dos artigos, reunindo 84 países, com destaque para Estados Unidos, Argentina, Alemanha, França e Reino Unido. Os indicadores de impacto internacional revelam um conjunto significativo de documentos citantes da produção científica, formado prioritariamente por artigos publicados em periódicos estrangeiros utilizando o idioma inglês. Os documentos citantes internacionais representaram 77,8\% do total e procedem de 168 países, com destaque para Estados Unidos, China, Reino Unido e Alemanha. Os resultados revelam que as Ciências Biológicas da Universidade Federal do Rio Grande do Sul acompanham as tendências de publicação internacional e integram-se às pesquisas realizadas no contexto global. Entretanto, a internacionalização da produção científica não ocorre de forma regular nos aspectos de difusão, colaboração e impacto internacional.

Palavras-chave: Bibliometria. Ciências Biológicas. Colaboração científica. Internacionalização. Produção científica. Universidade Federal do Rio Grande do Sul.

\begin{abstract}
The aim of the article was to analyze the internationalization of scientific publications in Biological Sciences at the Universidade Federal do Rio Grande do Sul from 2000 to 2011 in journals indexed by Web of Science. This is a bibliometric study and internationalization was examined under three main dimensions: international diffusion, collaboration and impact. The international diffusion indicators show strong preference for foreign journals and the English language. The articles were published in journals from 37 different countries and the percentage of documents published in foreign journals was $81.9 \%$. The international collaboration indicators reveal the prevalence of co-authorship in the publication of research results. International collaboration occurred in $22.4 \%$ of articles with the collaboration of

\footnotetext{
1 Artigo elaborado a partir da dissertação de mestrado de D.M. SANTIN, intitulada "Internacionalização da produção científica em Ciências Biológicas da UFRGS: 2000-2011". Universidade Federal do Rio Grande do Sul, 2013.

2 Universidade Federal do Rio Grande do Sul, Instituto de Ciências Básicas da Saúde, Biblioteca. R. Sarmento Leite, 500, Centro Histórico, 90050-170, Porto Alegre, RS, Brasil. Correspondência para/Correspondenceto: D.M.SANTIN. E-mail:<dirce.santin@ufrgs.br>.

3 Universidade Federal do Rio Grande do Sul, Faculdade de Biblioteconomia e Comunicação, Departamento de Ciências da Informação. Porto Alegre, RS, Brasil.
}

Recebido em 16/4/2014, reapresentado em 19/8/2014 e aceito para publicação em 29/9/2014.
\end{abstract}


84 countries, particularly the United States, United Kingdom Argentina, Germany and France. The international impact indicators reveal a significant set of citing documents of scientific production, primarily consisting of articles published in foreign journals in English. International citing documents accounted for $77.8 \%$ from 168 different countries, particularly from the United States, United Kingdom, China and Germany. The results reveal that Biological Sciences at Universidade Federal do Rio Grande do Sul follow the trends of international publication and are in agreement with research conducted throughout the world. However, internationalization of scientific production does not occur regularly considering international diffusion, collaboration and impact.

Keywords: Bibliometrics. Biological Sciences. Scientific collaboration. Internationalization. Scientific production. Universidade Federal do Rio Grande do Sul.

\section{Introdução}

A produção científica das universidades revela sua capacidade de cumprir uma de suas funções mais fundamentais: a criação de novos conhecimentos. A divulgação dos conhecimentos contribui para a internacionalização da ciência e das universidades. A tendência de internacionalização, observada em diversos países desde o final da década de 1980, ganhou força na virada do milênio e passou a integrar a preocupação dos estados e universidades brasileiras. A internacionalização tem-se estabelecido como estratégia de fortalecimento institucional, constituindo-se num processo para a melhoria da qualidade das atividades de ensino e pesquisa e num instrumento de projeção internacional das instituições (Sebastián, 2011).

A internacionalização da ciência e das universidades se expressa de diversas formas, que podem ser avaliadas com base em diferentes indicadores. Dentre as dimensões internacionais estão os resultados da atividade científica, representados por diversos elementos, como o conhecimento gerado a partir das atividades de investigação científica. A produção científica constitui um dos principais aspectos da internacionalização, pois demonstra a capacidade de países e instituições de produzir conhecimentos relevantes para a comunidade científica internacional. Consiste, também, num dos principais critérios de avaliação das universidades em índices nacionais e internacionais, considerando aspectos como visibilidade em bases de dados internacionais, colaboração internacional e impacto das citações recebidas de autores estrangeiros.

A Universidade Federal do Rio Grande do Sul (UFRGS) integra o grupo de universidades brasileiras mais bem avaliadas nos últimos anos pelos índices nacionais, tendo figurado como a melhor universidade do País na avaliação do Índice Geral de Cursos do Ministério da
Educação em 2012 e 2013 (Brasil, 2012, 2013), e entre as quatro melhores universidades brasileiras no Ranking Universitário Folha, também em 2012 e 2013 (Folha de São Paulo, 2012, 2013). No cenário internacional, destaca-se a avaliação do Academic Ranking of World Universities, em que a UFRGS classificou-se entre as 500 melhores universidades do mundo nas avaliações anuais de 2008 a 2013 (Academic Ranking of World Universities, 2013).

As Ciências Biológicas constituem uma das áreas mais consolidadas da UFRGS em ensino e pesquisa e uma das mais importantes na produção científica institucional. A área apresenta estrutura complexa, composta por dois institutos com três cursos de graduação, dez programas de Pós-Graduação, um centro de estudos e quatro órgãos auxiliares, além das contribuições do Hospital de Clínicas de Porto Alegre e dos mais de 120 grupos de pesquisa nas diversas subáreas.

No Brasil, a área constitui-se num dos campos mais sólidos em relação à pesquisa científica, com resultados de forte impacto social, cultural e econômico (Schwartzman, 2001). Seu desenvolvimento evidencia-se pela representatividade dos grupos de pesquisa e programas de pós-graduação, além dos índices elevados de produção científica. Os grupos de pesquisa totalizam 3 108, e correspondem a 11,3\% dos 27523 grupos nacionais (Conselho Nacional de Desenvolvimento Científico e Tecnológico, 2010). Na Pós-Graduação, são 258 programas com mestrado e doutorado, correspondendo a 13,9\% dos 1861 programas nacionais (Coordenação de Aperfeiçoamento de Pessoal de Nível Superior, 2014). A publicação de artigos completos de circulação nacional na área totalizou 38552 em 2010 (11\% dos artigos brasileiros), enquanto os artigos completos de circulação internacional somaram 84397 (25\% do total nacional) (Conselho Nacional de Desenvolvimento Científico e Tecnológico, 2010). 
As Ciências Biológicas brasileiras são tradicionalmente ligadas ao contexto internacional, tanto pelas expedições pioneiras de pesquisadores estrangeiros, quanto pelo intercâmbio e pela prática de divulgação internacional dos resultados das pesquisas. Estudos anteriores revelam a preferência dos pesquisadores da área pela publicação dos resultados em periódicos internacionais (Meneghini \& Fonseca, 1990; Melo et al. 2011). A internacionalização das publicações também é um padrão da área, cujos temas não estão, necessariamente, circunscritos a um espaço geográfico, cultural e histórico (Santos, 2003).

Este artigo analisa as dimensões internacionais da produção científica em Ciências Biológicas da UFRGS, em termos de artigos publicados em revistas indexadas pela Web of Science (WoS) no período de 2000 a 2011. A internacionalização é examinada sob três dimensões principais, com base na classificação proposta pelo Manual de Santiago (Red de Indicadores de Ciencia y Tecnología Iberoamericana e Interamericana, 2007): a difusão internacional, caracterizada pela publicação dos artigos em periódicos internacionais e em idioma inglês; a colaboração internacional, caracterizada pela coautoria com autores de outros países; e o impacto internacional das publicações, caracterizado pela proporção de documentos internacionais citantes dos artigos.

\section{Procedimentos metodológicos}

A pesquisa caracteriza-se como um estudo bibliométrico de nível médio de agregação, dedicado à análise da produção científica da grande área de uma instituição. Com abordagem quantitativa, analisa os artigos em Ciências Biológicas da UFRGS publicados em periódicos indexados pela WoS no período de 2000 a 2011. A opção pelos artigos considera a relevância das publicações na área (Meadows, 1999), sua cobertura na indexação da WoS (Red de Indicadores de Ciencia y Tecnología Iberoamericana e Interamericana, 2007) e a publicação em periódicos científicos, que constituem o meio de comunicação mais aceito na ciência (Stumpf, 2005).

A avaliação quantitativa da ciência, adotada nesta pesquisa, orienta-se pelo uso de indicadores bibliométricos e constitui a área chamada Bibliometria ou Ciento- metria. A área compreende os estudos da ciência baseados em fontes de arquivo, incluindo estudos de citação, colaboração e produtividade científica, entre outros (Velho, 1990). A avaliação quantitativa é considerada uma tendência mundial, configurando-se recurso essencial à construção de indicadores e à definição de estratégias regionais e institucionais, além de servir à avaliação das políticas científicas.

A principal fonte de dados foi a base de dados WoS. Sua utilização baseia-se no uso amplo pela comunidade científica como fonte de dados para os estudos bibliométricos e na sua extensa cobertura de periódicos internacionais e regionais, especialmente nas Ciências Naturais (Red de Indicadores de Ciencia y Tecnología Iberoamericana e Interamericana, 2007). As fontes complementares foram a Plataforma Lattes e as páginas de instituições e países colaboradores e/ou citantes. 0 tratamento dos dados foi realizado com os softwares Microsoft Excel, BibExcel e Philcarto.

O corpus da pesquisa foi formado por dois conjuntos de dados. O primeiro conjunto, utilizado para as análises de difusão e colaboração internacional, foi coletado em abril de 2013. A coleta ocorreu pelo campo Busca Avançada, utilizando a estratégia de busca por endereço da instituição (AD), limitada a 33 categorias de assunto da WoS (WC), ao período de 2000 a 2011 (PY), ao tipo de documento artigo e ao Science Citation Index (SCI). Os resultados de busca foram exportados da base no formato completo, utilizando a opção Texto sem Formatação, em grupos de 500. Após a limpeza dos dados, os resultados totalizaram 5168 artigos.

As 33 categorias de assunto da WoS utilizadas na pesquisa foram: Anatomia \& Morfologia; Andrologia; Biodiversidade \& Conservação; Biofísica; Biologia; Biologia Celular; Biologia do Desenvolvimento; Biologia Evolutiva; Biologia Marinha \& de Água Doce; Biologia Matemática \& Computacional; Biologia Reprodutiva; Bioquímica \& Biologia Molecular; Biotecnologia \& Microbiologia Aplicada; Ciências Ambientais; Ciências das Plantas; Doenças Infecciosas; Ecologia; Endocrinologia \& Metabolismo; Entomologia; Farmacologia \& Farmácia; Fisiologia; Genética \& Hereditariedade; Imunologia; Métodos de Pesquisa Bioquímica; Micologia; Microbiologia; Neurociências; Ornitologia; Parasitologia; Patologia; Toxicologia; Virologia; e Zoologia. A seleção das categorias 
considerou a estrutura das Ciências Biológicas na Tabela de Áreas do Conhecimento do Conselho Nacional de Desenvolvimento Científico e Tecnológico (CNPq) e a configuração atual da área na UFRGS.

O segundo conjunto de dados, utilizado para as análises de impacto internacional, foi formado por 40600 documentos e incluiu as publicações que atribuíram citações aos artigos até junho de 2013. A coleta dos dados ocorreu a partir do ícone Criar Relatório de Citações, disponível na tela de resultados da busca anterior, repetida em junho de 2013. O Relatório de Citações permitiu identificar os documentos citantes e exportar seus registros da base. Posteriormente, os registros foram reunidos em dois arquivos, de acordo com o conjunto de dados a que pertenciam, e os nomes de autores e instituições foram normalizados, de modo a reunir entradas de nomes equivalentes e garantir confiabilidade aos resultados do estudo.

A internacionalização da produção científica foi analisada e é apresentada a seguir através de três grupos de indicadores: (1) difusão internacional, caracterizada pela publicação dos artigos em periódicos internacionais e em inglês; (2) colaboração internacional, representada pela coautoria com autores e instituições de outros países; (3) impacto internacional das publicações, representado pela proporção de documentos citantes estrangeiros em relação ao total e pela origem geográfica dos citantes internacionais.

\section{Resultados}

A produção científica em Ciências Biológicas da UFRGS apresentou crescimento significativo, com média de $11,6 \%$ ao ano, correspondendo a cerca de $30,0 \%$ dos artigos publicados pela instituição no período. A evolução da produção científica revela a intensa atividade da área e está associada a diversos fatores, como a ampliação do número de cursos de pós-graduação e de professores a eles vinculados e a criação de novos grupos de pesquisa, além de fatores externos, como as políticas científicas nacionais e a ampliação da cobertura da WoS no início do milênio, que resultou em maior número de periódicos brasileiros indexados na base. Os dados confirmam a importância dos artigos da área no contexto institucional e contribuem para manter a UFRGS entre as cinco instituições brasileiras mais produtivas na WoS.

\section{Difusão internacional}

A difusão internacional foi objeto do primeiro grupo de indicadores. A indexação dos periódicos em bases de dados internacionais é característica básica de internacionalização da produção científica (Red de Indicadores de Ciencia y Tecnología Iberoamericana e Interamericana, 2007), de modo que todos os artigos contemplados na pesquisa podem ser considerados internacionais. Entretanto, optou-se por ampliar a análise e avaliar a difusão internacional pela proporção de artigos publicados em periódicos estrangeiros. Nessa perspectiva, a produção científica em Ciências Biológicas da UFRGS publicada entre 2000 e 2011 apresenta elevado alcance internacional, pois foi veiculada em 931 periódicos, dos quais 898 estrangeiros $(96,5 \%)$ e 33 brasileiros (3,5\%). Já a proporção de artigos publicados em periódicos estrangeiros foi de $81,9 \%$, contra $18,1 \%$ publicados em periódicos nacionais.

A difusão internacional também foi analisada em relação à distribuição geográfica dos periódicos e seus artigos pelos países de origem das publicações. Os resultados revelam que os principais países responsáveis pela publicação dos periódicos que veiculam os artigos são Estados Unidos (EUA), com 341 títulos (36,7\%); Reino Unido, com 249 títulos (26,8\%); Holanda, com 123 títulos (13,3\%); e Brasil, com 33 títulos (3,5\%). A concentração das publicações em países desenvolvidos foi destacada por estudos anteriores, que apontam para a existência de uma zona central que se sobrepõe na publicação dos periódicos, além dos índices e bases de dados que lhe dão acesso (Mueller \& Oliveira, 2003). A concentração ocorre também em relação aos editores. Supõe-se, por exemplo, que os altos índices registrados pela Holanda neste estudo reforcem a forte presença no meio científico do grupo internacional Elsevier, sediado naquele país.

Quando analisada a distribuição dos artigos por país de publicação, EUA e Reino Unido mantêm-se nas primeiras posições, publicando 1627 (31,5\%) e 1091 $(21,1 \%)$ artigos, respectivamente, e Brasil e Holanda invertem suas posições, publicando 934 (18,1\%) e 816 artigos (15,8\%). Em seguida destacam-se Alemanha, com 174 artigos (3,4\%); Irlanda, com 126 artigos (2,4\%); e Argentina, com 66 artigos (1,3\%). O mapa (Figura 1) demonstra a intensidade de publicação entre os diversos países. 


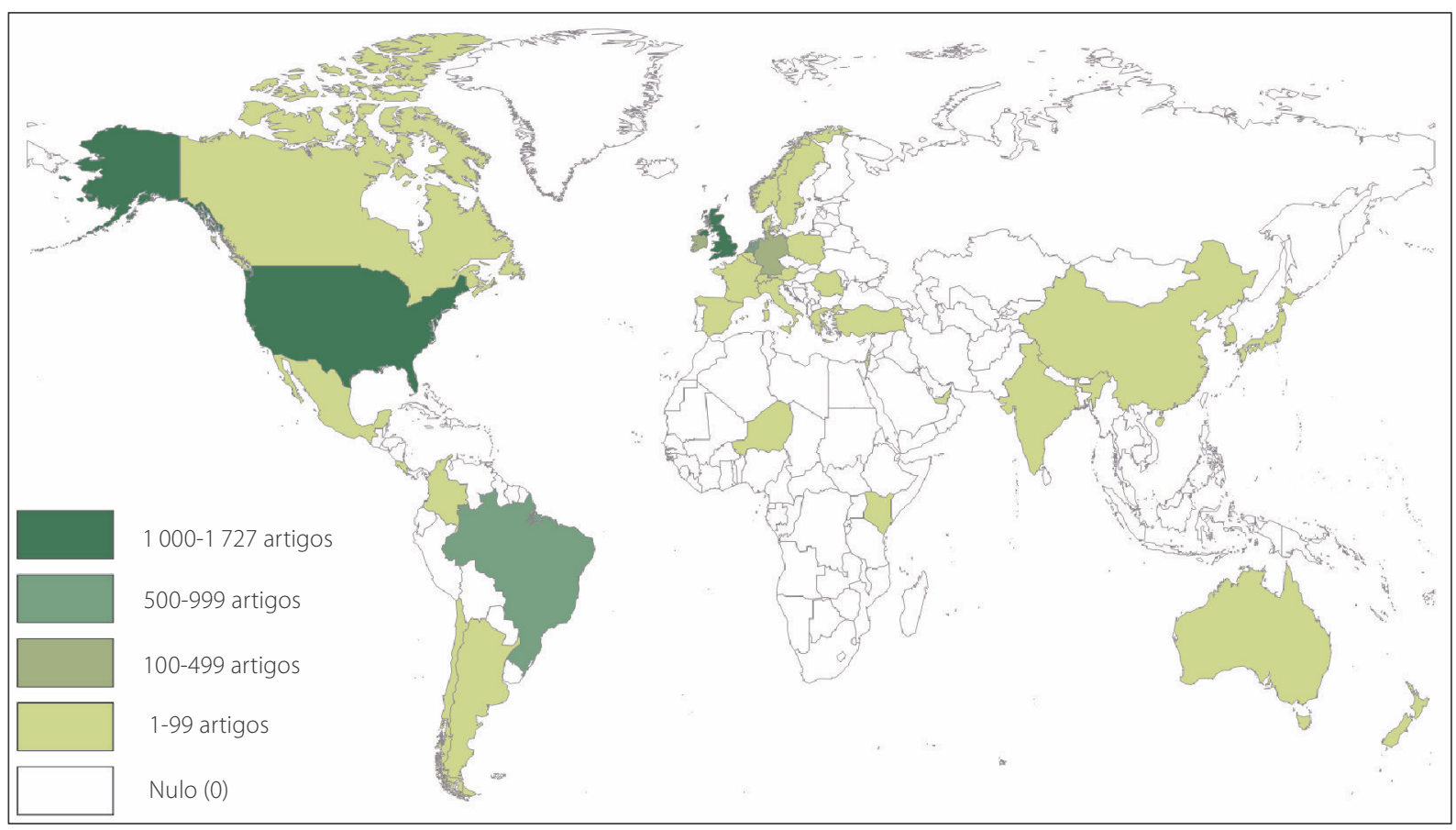

Figura 1. Difusão internacional dos artigos em Ciências Biológicas da UFRGS entre os países publicadores (2000-2011).

Fonte: Elaborada pelas autoras (2014).

A publicação dos artigos ocorreu em 37 países, incluindo o Brasil. O uso do idioma inglês foi elevado e ocorreu em $94,8 \%$ dos artigos. A situação reforça a preferência dos pesquisadores da área pela publicação de artigos em periódicos internacionais (Meneghini \& Fonseca, 1990; Melo et al., 2011) e no idioma inglês (Meadows, 1999), permitindo avaliar que a produção científica em Ciências Biológicas da UFRGS é fortemente internacionalizada no aspecto da difusão, pela publicação em periódicos internacionais e no idioma inglês.

\section{Colaboração internacional}

A colaboração internacional constituiu objeto do segundo grupo de indicadores, destinado à análise da colaboração com outros países. A coautoria predomina na publicação dos resultados das pesquisas, representando um padrão nas Ciências Biológicas da UFRGS. Dentre os 5168 artigos, apenas 40 (0,8\%) foram publicados em autoria única. A autoria múltipla foi registrada em 5128 artigos (99,2\%). Desse conjunto, 1158 artigos (22,4\%) foram produzidos em colaboração internacional, enquanto 3970 (76,8\%) registraram colaboração entre autores nacionais.

A proporção de colaboração internacional revela-se inferior ao índice observado por Leta e Chaimovich (2002) na produção científica brasileira publicada em 1990, quando a colaboração internacional ocorreu em 26,7\% das publicações; ao índice identificado por Glänzel (2001) na produção científica brasileira publicada de 1995 a 1996, cujo percentual de colaboração internacional foi de 41,7\%; e ao índice observado por Vanz (2009) na produção científica brasileira publicada de 2004 a 2006, em que a colaboração internacional foi 30,3\%.

Entretanto, o índice de 22,4\% aproxima-se da média de colaboração internacional identificada na produção científica global. Glänzel e Schubert (2004) observaram que $17,0 \%$ dos artigos do mundo todo publicados em 2000 foram produzidos por autores de dois ou mais países, enquanto Leydesdorff e Wagner (2008) identificaram um índice de colaboração internacional de 23,3\% na produção científica mundial publicada em 2005. Em estudo recente, Adams (2013) observou um decréscimo na colaboração internacional em países em 
desenvolvimento, como Brasil, China, Índia e Coreia do Sul, entre os anos 1981 e 2012. A situação é contrária em países desenvolvidos, como EUA, Reino Unido, Alemanha, França, Holanda e Suiça, onde a colaboração internacional alcança índices cada vez maiores.

Em relação ao nível de colaboração internacional, que considera o número de países envolvidos na produção científica, observou-se a prevalência da colaboração bilateral, registrada em 912 artigos (78,8\%); seguida da colaboração trilateral, em 140 artigos (12,1\%); e da colaboração multilateral, em 106 artigos (9,1\%). A situação acompanha a tendência de colaboração bilateral verificada na produção científica brasileira (Vanz, 2009) e indica, ainda que em menor grau, a participação das Ciências Biológicas da UFRGS na chamada Big Science, que se caracteriza pela complexidade e pelo envolvimento de grande número pesquisadores em pesquisas de interesse global (Price, 1976).

Os artigos publicados em colaboração internacional foram produzidos por autores de 84 países, além do Brasil. A distribuição dos artigos produzidos com os principais países colaboradores permite observar os parceiros mais frequentes e inferir sobre sua importância na internacionalização da produção científica (Tabela 1). Destacam-se EUA, Argentina, Alemanha, França e Reino Unido, além de outros parceiros que pontuaram dez ou mais artigos em colaboração com a UFRGS.

A coautoria das Ciências Biológicas da UFRGS com autores americanos supera significativamente os índices identificados para a produção científica brasileira em estudos anteriores (Leta \& Chaimovich, 2002; Vanz, 2009). A forte participação dos EUA pode ser associada à proeminência daquele país como zona central da ciência e à liderança exercida no campo científico (Mueller \& Oliveira, 2003), embora também indique certa dependência na produção científica. Na perspectiva da internacionalização da ciência, a colaboração com os EUA pode elevar a visibilidade das publicações e ampliar seu impacto na comunidade científica internacional, embora o processo de internacionalização também exija diversificação de parceiros e simetria nas relações de colaboração (Olmeda-Gómez et al., 2009; Sebastián, 2011).

A segunda posição, ocupada pela Argentina, também acompanha o padrão de colaboração estabelecido na produção científica brasileira (Glänzel et al.,
Tabela 1. Países colaboradores na produção científica em Ciências Biológicas da UFRGS (2000-2011).

\begin{tabular}{|c|c|c|c|}
\hline \multicolumn{2}{|c|}{ País } & \multirow{2}{*}{ Artigos } & \multirow{2}{*}{$\begin{array}{c}\% \\
18,3\end{array}$} \\
\hline 1 & EUA & & \\
\hline 2 & Argentina & 201 & 3,9 \\
\hline 3 & Alemanha & 187 & 3,6 \\
\hline 4 & França & 174 & 3,4 \\
\hline 5 & Reino Unido & 169 & 3,3 \\
\hline 6 & Espanha & 133 & 2,6 \\
\hline 7 & Canadá & 120 & 2,3 \\
\hline 8 & Itália & 99 & 1,9 \\
\hline 9 & Austrália & 93 & 1,8 \\
\hline 10 & Uruguai & 67 & 1,3 \\
\hline 11 & Holanda & 63 & 1,2 \\
\hline 12 & Portugal & 51 & 1,0 \\
\hline 13 & Suiça & 50 & 1,0 \\
\hline 14 & Japão & 46 & 0,9 \\
\hline 15 & Chile & 44 & 0,8 \\
\hline 16 & Finlândia & 38 & 0,7 \\
\hline 17 & México & 36 & 0,7 \\
\hline 18 & Colômbia & 32 & 0,6 \\
\hline 19 & Áustria & 31 & 0,6 \\
\hline 20 & Suécia & 29 & 0,6 \\
\hline 21 & Dinamarca & 24 & 0,5 \\
\hline 22 & Rússia & 20 & 0,4 \\
\hline 23 & África do Sul & 20 & 0,4 \\
\hline 24 & Polônia & 16 & 0,3 \\
\hline 25 & Peru & 15 & 0,3 \\
\hline 26 & Israel & 14 & 0,3 \\
\hline 27 & Bélgica & 13 & 0,2 \\
\hline 28 & Cuba & 12 & 0,2 \\
\hline 29 & Taiwan & 11 & 0,2 \\
\hline 30 & China & 11 & 0,2 \\
\hline 31 & Eslovênia & 11 & 0,2 \\
\hline 32 & Costa Rica & 10 & 0,2 \\
\hline 33 & República Tcheca & 10 & 0,2 \\
\hline
\end{tabular}

Fonte: Elaborada pelas autoras (2014).

Nota: Percentual calculado em relação ao total de artigos: 5168.

2006; Vanz, 2009). Em sequência destacam-se os países europeus, cuja predominância foi observada por Leta e Chaimovich (2002) na produção científica brasileira publicada de 1981 a 1999. A colaboração com países do hemisfério sul tende a ser menor, podendo ser associada a parcerias estabelecidas entre pesquisadores e convênios firmados entre as nações.

Outro aspecto da colaboração internacional refere-se às instituições estrangeiras colaboradoras. Foram identificadas 951 instituições coautoras procedentes de outros países. O número é elevado e demonstra a heterogeneidade das relações de colaboração estabelecidas pela Universidade, embora também possa 
indicar a necessidade de convênios e parcerias mais estáveis com as principais parceiras.

A Universidad de Buenos Aires é a principal instituição estrangeira colaboradora, com 111 artigos em coautoria (2,1\%). A situação revela a importância da instituição na produção dos artigos e reforça a relevância da colaboração estabelecida pela UFRGS com a Argentina. Os EUA, por sua vez, reúnem o maior número de instituições entre as principais colaboradoras, assumindo as posições seguintes com a University of California, com 69 artigos (1,3\%); a Harvard University, com 51 artigos $(1,0 \%)$; a University of North Carolina, com 44 artigos $(0,8 \%)$; e a University of Texas, com 44 artigos (0,8\%). A colaboração com instituições europeias também é bastante significativa, com destaque para Reino Unido (4), Espanha (3), França (3), Alemanha (3), Finlândia (2) e Portugal (2).

\section{Impacto internacional}

Os indicadores de impacto internacional encerram a análise da internacionalização da produção cientí- fica. As características gerais revelam um volume significativo de documentos citantes, com média de crescimento de 48,9\% ao ano; a hegemonia dos artigos entre os diversos tipos de documentos citantes; e a forte prevalência do inglês como idioma das publicações que atribuem citações aos artigos.

A procedência dos principais periódicos responsáveis pelas citações também indica a internacionalidade do impacto alcançado. No conjunto de 69 títulos que publicaram 70 ou mais documentos citantes, a proporção de periódicos estrangeiros é de 88,4\% (61 títulos), enquanto os brasileiros representam 11,6\% (oito títulos). Destacam-se os periódicos PLoS One, com 617 documentos (1,5\%); Brain Research, com 289 documentos $(0,7 \%)$; Behavioural Brain Research, com 277 documentos $(0,7 \%)$; Neurochemical Research, com 265 documentos $(0,7)$; e Neuroscience, com 237 documentos (0,6\%).

O número de documentos internacionais citantes dos artigos em Ciências Biológicas da UFRGS foi 31 580, enquanto o total de citantes nacionais foi 9020 . Dessa forma, o impacto internacional corresponde a $77,8 \%$ dos documentos citantes, contra 22,2\% de citantes nacionais.

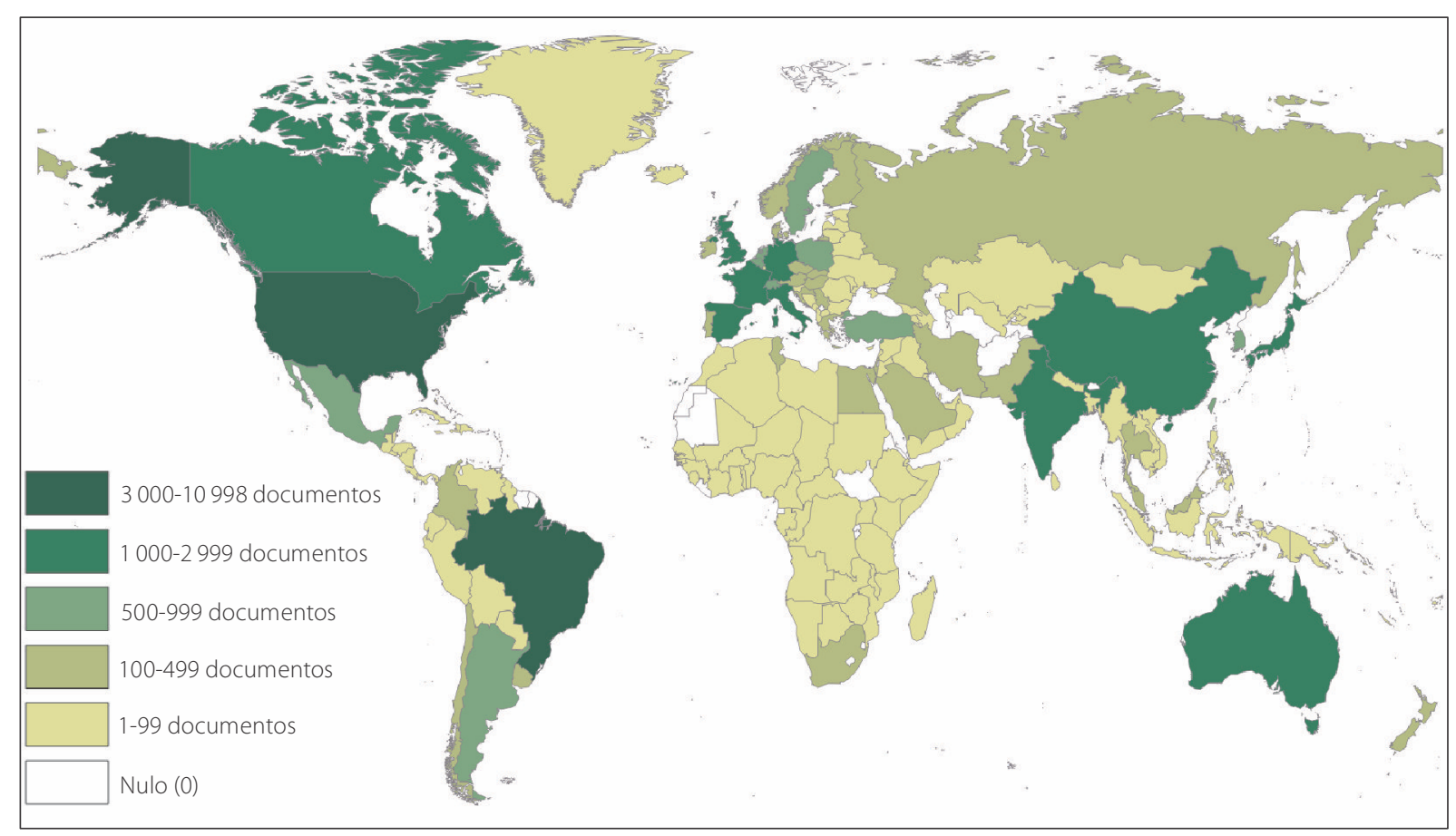

Figura 2. Impacto internacional dos artigos em Ciências Biológicas da UFRGS nos países citantes (2000-2013).

Fonte: Elaborada pelas autoras (2014). 
Dentre os documentos internacionais, 29602 (72,9\%) são originários de autores exclusivamente estrangeiros, enquanto 1978 (4,9\%) foram produzidos por autores de outros países em colaboração com o Brasil.

Entre os principais países citantes destacam-se: Brasil, com 10998 documentos (27,1\%); EUA, com 9291 (22,9\%); China, com 2545 (6,3\%); Reino Unido, com 2484 (6,1\%); e Alemanha, com 2207 documentos (5,4\%). O volume de documentos citantes procedentes desses países pode sugerir o reconhecimento da atividade científica da área na Universidade, mas também está associado à liderança científica dos países, além da colaboração estabelecida com a UFRGS na produção dos artigos. Os artigos foram citados em publicações de autores de 168 países, incluindo o Brasil, conforme indicado na Figura 2.

O mapa possibilita a visualização da distribuição geográfica dos documentos citantes no globo, com ênfase nos principais países de origem dos autores citantes. A imagem revela a amplitude do impacto obtido pelos artigos e a diversidade geográfica dos documentos citantes. O impacto em grande número de países pode ampliar a difusão internacional dos artigos por meio das referências citadas nas publicações (Red de Indicadores de Ciencia y Tecnología Iberoamericana e Interamericana, 2007), assim como a colaboração internacional expressa na coautoria dos artigos tende a resultar em maior impacto no meio científico internacional (Adams, 2013). Avalia-se, portanto, que as dimensões internacionais da produção científica complementam-se e fortalecem-se mutuamente, gerando um fluxo contínuo que se renova em novas publicações, cujo uso pela comunidade científica pode ser comprovado pelas citações recebidas de outras publicações.

Outro ponto analisado em relação ao impacto internacional foram as principais instituições estrangeiras de filiação dos autores. $O$ aspecto pode indicar a importância e o prestígio dos artigos junto às instituições internacionais, além de sugerir tendências de concentração e similaridade das pesquisas (Vanz \& Caregnato, 2003). As principais instituições estrangeiras citantes são originárias, primordialmente, dos EUA, que ocupam as primeiras posições, da Inglaterra, da Austrália e do Canadá, além de países como Argentina, Espanha, França e Holanda. A University of California ocupa posição de destaque entre as estrangeiras citantes, com 983 documentos (2,4\%); seguida da Harvard University, com 506 (1,1\%); da University of Texas, com 439 (1,1\%); da Chinese Academy of Sciences, com 427 (1,1\%); e da Universidad de Buenos Aires, com 326 documentos (0,8\%).

O impacto observado em relação aos países amplia-se na análise de instituições, revelando baixa concentração. A característica revela a existência de grande dispersão entre as instituições citantes, pois algumas registram autoria em grande número de documentos, enquanto muitas outras registram participação menor e, por vezes, insignificante. A situação acompanha a Lei de Bradford, que permite estabelecer o núcleo e as áreas de dispersão num conjunto de periódicos (Pinheiro, 1983) e a regra 80/20, pela qual é possível supor que cerca de 20\% das instituições são responsáveis por cerca de 80\% dos documentos citantes (Barabási, 2009). Por outro lado, a situação reforça o aspecto da diversidade geográfica (e institucional), essencial aos processos de internacionalização da ciência, de modo que pode ser considerada altamente positiva nesse contexto.

\section{Conclusão}

A internacionalização da ciência ganhou importância nas últimas décadas e tornou-se tema estratégico no planejamento e na avaliação das políticas científicas nacionais e institucionais. O protagonismo das dimensões internacionais evidencia-se neste estudo, que destacou a internacionalização da pesquisa em Ciências Biológicas da UFRGS a partir dos aspectos de difusão, colaboração e impacto internacional das publicações. As dimensões internacionais observadas na produção científica refletem aspectos específicos, que se complementam e fortalecem entre si. A análise conjunta dos aspectos relativos à difusão, à colaboração e ao impacto internacional representa o caminho metodológico adequado para a compreensão da internacionalização da produção científica de países e instituições.

Os indicadores de internacionalização operacionalizados na pesquisa demonstram que as Ciências Biológicas da UFRGS acompanham as tendências de publicação internacional e integram-se às pesquisas realizadas no contexto global. Entretanto, a internacionalização não ocorre de forma regular nas diversas dimensões. Enquanto a proporção de artigos publicados 
em periódicos estrangeiros foi de 81,9\% e a proporção de documentos internacionais citantes dos artigos foi de $77,8 \%$, a colaboração internacional ocorreu em apenas 22,4\% das publicações. Compreende-se que a situação não reduz a importância das pesquisas e a capacidade objetiva da área em projetar os resultados da ciência no meio científico internacional. Avalia-se, entretanto, que políticas e ações voltadas ao fortalecimento da colaboração internacional assumem importância estratégica no processo de internacionalização, podendo conferir à UFRGS o almejado status de instituição de classe mundial.

Embora restrito à análise da internacionalização da produção científica publicada na forma de artigos e indexada na WoS, este estudo contribui para o conhecimento das dimensões internacionais da pesquisa em Ciências Biológicas da UFRGS. Os resultados fornecem informações sobre a projeção internacional e podem servir ao aperfeiçoamento das políticas de internacionalização da Universidade. Não obstante, é preciso considerar que a preocupação com a internacionalização das universidades ampliou-se nas últimas décadas no Brasil. A configuração não é diferente na UFRGS, de modo que as estratégias e políticas mais recentes poderão refletir-se mais fortemente na internacionalização da produção científica institucional nos próximos anos.

\section{Referências}

Academic Ranking of World Universities. As 500 melhores universidades mundiais. 2013. Disponível em: <http://www. shanghairanking.com/ARWU2013.html>. Acesso em: 15 mar. 2014.

Adams, J. Collaborations: The fourth age of research. Nature, v.497, n.7451, p.557-560, 2013.

Barabási, A-L. Linked (conectado): a nova ciência dos networks. São Paulo: Leopardo, 2009.

Brasil. Ministério da Educação. Instituto Nacional de Estudos e Pesquisas Educacionais Anísio Teixeira. Índice geral de cursos. Brasília: Inep, 2012. Disponível em: < http://portal.inep.gov.br/ indice-geral-de-cursos>. Acesso em: 15 mar. 2014.

Brasil. Ministério da Educação. Instituto Nacional de Estudose Pesquisas Educacionais Anísio Teixeira. Índice geral de cursos. Brasília: Inep, 2013. Disponível em: <http://portal.inep.gov.br/ indice-geral-de-cursos>. Acesso em: 15 mar. 2014.

Conselho Nacional de Desenvolvimento Científico e Tecnológico. Diretório de grupos de pesquisa no Brasil. Brasília, 2010. Disponível em: <http://dgp.cnpq.br/censos/>. Acesso em: 28 mar. 2014.

Coordenação de Aperfeiçoamento de Pessoal de Nível Superior. Cursos recomendados e reconhecidos. Brasília, 2014. Disponível em: <http://capes.gov.br/avaliacao/cursosrecomendados-e-reconhecidos>. Acesso em: 29 mar. 2014.

Folha de São Paulo. Ranking universitário Folha. São Paulo, 2012. Disponível em: <http://ruf.folha.uol.com.br/2012/>. Acesso em: 15 mar. 2014.

Folha de São Paulo. Ranking universitário Folha. São Paulo, 2013. Disponível em: <http://ruf.folha.uol.com.br/2013/>. Acesso em: 15 mar. 2014.

Glänzel, W. National characteristics in international scientific co-authorship relations. Scientometrics, v.51, n.1, p.69-115, 2001.
Glänzel, W.; Leta, J.; Thijs, B. Science in Brazil: A macro-level comparative study. Scientometrics, v.67, n.1, p.67-86, 2006.

Glänzel, W.; Schubert, A. Analyzing scientific networks through co-authorship. In: Moed, H.F.; Glänzel, W.; Schmoch, U. (Ed.). Handbook of quantitative science and technology research. New York: Kluwer Academic Publishers, 2004. p.257-276.

Leta, J.; Chaimovich, $\mathrm{H}$. Recognition and international collaboration. Scientometrics, v.53, n.3, p.325-335, 2002.

Leydesdorff, L.; Wagner, C. International collaboration in science and the formation of a core group. Journal of Informetrics, v.2, n.4, p.317-325, 2008.

Meadows, A.J. A comunicação científica. Brasília: Briquet de Lemos, 1999.

Melo, P.L.C.; Mugnaini, R.; Leta, J. A new indicator for international visibility: Exploring Brazilian scientific community. Scientometrics, v.88, n.1, p.311-319, 2011.

Meneghini, R.; Fonseca, L. Índices alternativos de avaliação da produção científica em Bioquímica no Brasil. Ciência e Cultura, v.42, n.9, p.629-645, 1990.

Mueller, S.P.M.; Oliveira, H.V. Autonomia e dependência na produção da ciência: uma base conceitual para estudar relações na comunicação científica. Perspectivas em Ciência da Informação, v.8, n.1, p.58-65, 2003.

Olmeda-Gómez, C. et al. Visualization of scientific coauthorship in Spanish universities: From regionalization to internationalization. Aslib Proceedings, v.61, n.1, p.83-100, 2009.

Pinheiro, L.V.R. Lei de Bradford: uma reformulação conceitual. Ciência da Informação, v.12, n.2, p.59-80, 1983.

Price, J.D.S. O desenvolvimento da ciência: análise histórica, filosófica, sociológica e econômica. Rio de Janeiro: LTC, 1976.

Red de Indicadores de Ciencia y Tecnología Iberoamericana e Interamericana. Manual de indicadores de internacionalización 
de la ciencia y la tecnología. Buenos Aires, 2007. Disponível em: <http://www.oei.es/salactsi/manual_santiago.pdf>. Acesso em: 20 mar. 2014.

Santos, C. M. Tradições e contradições da pós-graduação no Brasil. Educação \& Sociedade, Campinas, v.24, n.83, p.627-641, 2003.

Schwartzman, S. Um espaço para a ciência: a formação da comunidade científica no Brasil. Brasília: Ministério da Ciência e Tecnologia, 2001.

Sebastián, J. Dimensiones y métrica de la internacionalización de las universidades. Universidades, v.51, p.3-16, 2011.
Stumpf, I.R.C. Avaliação de originais nas revistas científicas. In: Ferreira, S.M.P.; Targino, M.G. (Org.). Preparação de revistas científicas. São Paulo: Reichmann, 2005. p.103-121.

Vanz, S.A.S. As redes de colaboração científica no Brasil: (2004-2006). 2009. Tese (Doutorado em Comunicação e Informação) - Universidade Federal do Rio Grande do Sul, Porto Alegre, 2009.

Vanz, S.A.S.; Caregnato, S.E. Estudos de citação: uma ferramenta para entender a comunicação científica. Em Questão, v.9, n.2, p.295-307, 2003.

Velho, L. Indicadores científicos: em busca de uma teoria. Interciencia, v.15, n.3, p.139-145, 1990. 
